\title{
Complete determination of the elastic moduli of a-quartz under hydrostatic pressure up to 1 GPa: an ultrasonic study
}

\author{
Ernesto Calderon ${ }^{1}$, Michel Gauthier, Frédéric Decremps, Gérard Hamel, \\ Gérard Syfosse and Alain Polian
}

Physique des Milieux Denses, Institut de Minéralogie et de Physique des Milieux Condensés, CNRS, Université Pierre et Marie Curie-Paris 6, 140 rue de Lourmel, 75015 Paris, France

\begin{abstract}
Using the ultrasonic pulse-echo technique and a newly developed method to treat the data, all the elastic compliances of $\alpha$-quartz have been obtained as a function of hydrostatic pressure up to $1 \mathrm{GPa}$ with great accuracy. Fifteen independent measurements were performed in five directions of high symmetry $\left(X, Y, Z, Y^{\mathrm{t}}\right.$ and $\left.Z^{\mathrm{t}}\right)$. The pressure dependence of the six second-order elastic and two piezoelectric constants were deduced. The pressure derivatives of $c_{14}$ and $c_{44}$ are positive, whereas that of $c_{66}$ is negative, in contradiction to previously published results. Under ambient conditions, the linear and bulk moduli $B_{a 0} \biguplus 3.4(0.5) \mathrm{GPa}, B_{c 0} 13 Z 0(0.5) \mathrm{GPa}$ and $B_{0} 37.5(\Theta .2) \mathrm{GPa}$ calculated from our determination of the elastic tensor are in good agreement with the published values. However, we find a clear discrepancy with published values for the pressure derivative of the bulk modulus $B^{\mathrm{t}} 4 Z(0.5)$. From these results, the Born stability criteria have been calculated as a function of pressure and compared with previous results. Their behavior can help to explain the structural instability of $\alpha$-quartz at around $18 \mathrm{GPa}$.
\end{abstract}

(Some figures in this article are in colour only in the electronic version)

\section{Introduction}

$\mathrm{SiO}_{2}$ is one of the most abundant materials in the lithosphere. It exists in several crystalline structures. Among them $\alpha$-quartz is the stable one at ambient conditions. Because of this abundance and of the many possible applications using its mechanical, piezoelectrical, optical and chemical properties, it is one of the most studied compounds of the last 25 years [1-3]. Its crystal structure is well established [4] and has been thoroughly investigated

${ }^{1}$ Permanent address: Centro de Estudios de Semiconductores, Departamento de Fisica, Falculta de Ciencias, Universidad de Los Andes, Mérida, Venezuela. 
as a function of temperature [5, 6] and pressure [7-11]. At ambient temperature, $\boldsymbol{\alpha}$-quartz remains stable up to approximately $18 \mathrm{GPa}$. At higher pressure, several experimental results show a gradual amorphization, producing heterogeneous samples of coexisting crystalline and amorphous phases [8, 9]. In spite of the large number of theoretical [12-19] and experimental [8-11, 20-24] papers, the process of pressure-induced amorphization is not well understood. Several conflicting suggestions have been made to explain the mechanism of this transformation. Murashov [23] suggests that the phase transition at $20 \mathrm{GPa}$ is connected with the formation of a layered phase resembling the high-pressure phase of $\mathrm{GaPO}_{4}$. Alternating layers with an ordered distribution of cross linking bonds can be responsible for the appearance of signs of quartz superstructure in the experimental diffraction patterns of the compressed crystal. Kingma et al [8] show the existence of a metastable crystal-crystal transformation occurring prior to or during amorphization. Some authors propose that the structural instability might be correlated with the behavior under stress of a particular elastic modulus, i.e. amorphization would be a consequence of a mechanical instability [14] that can be observed through the variation of the Born stability criterion [25]. This criterion expresses the fact that any mechanical strain must increase the total mechanical energy of a system at equilibrium. Concerning this criterion, it seems to be necessary to emphasize that in crystals belonging to the $32,3 m$ and $\overline{3} m$ trigonal classes, the Born criterion gives rise to only two conditions [26]

$$
\begin{aligned}
B_{1} & =\left(c_{11}+c_{12}\right) c_{33}-2 c_{13}^{2} \\
& =2\left(\left(c_{11}-c_{66}\right) c_{33}-c_{13}{ }^{2}\right)>0 \\
B_{2} & =\left(c_{11}-c_{12}\right) c_{44}-2 c_{14}^{2} \\
& =2\left(c_{66} c_{44}-c_{14}{ }^{2}\right)>0 .
\end{aligned}
$$

In hexagonal crystals, $c_{14} \Theta$, so $B_{2}$ splits into two conditions: $c_{44}>0$ and $c_{66}>0$. The condition $c_{66}>0$ often written as $c_{11-\phi_{12}} \ngtr 0$ has no meaning in trigonal crystals. This last condition is called ' $B_{1}$ ' in the papers dealing with quartz, the correct $B_{1}$ (equation $(1 a)$ ) and $B_{2}$ (equation $(1 b)$ ) are denoted $B_{2}$ and $B_{3}$ in the literature, respectively.

The first criterion $B_{1}$ (equation (1a)) expresses that the sample volume cannot collapse upon compression as can be seen in the expression for the bulk and linear moduli.

$$
\begin{aligned}
B_{0}= & \frac{c_{33}\left(c_{11}+c_{12}\right)-2 c^{2}}{c_{11}+c_{12}+2 c_{33}-4 c_{13}} \\
= & \frac{B^{1}}{c_{11}+c_{12}+2 c_{33}-4 c_{13}} \\
B_{a 0}= & \frac{c_{33}\left(c_{11}+c_{12}\right)-2 c^{2}}{c_{33}-c_{13}}=\frac{B_{1}}{c_{33}-c_{13}} \\
B_{c 0}= & \frac{c_{33}\left(c_{11}+c_{12}\right)-2 c^{2}}{c_{11}+c_{12}-2 c_{13}} 3=\frac{B_{1}}{c_{11}+c_{12}-2 c_{13}} .
\end{aligned}
$$

No acoustic mode can produce the strain required by this criterion. On the contrary, $B_{2}$ (equation $(1 b)$ ) is associated with two different eigenvalues corresponding to different acoustic modes $[27,28]$. A decrease of the $B_{2}$ value under compression denotes the existence of a mechanical instability correlated with the presence of a soft acoustic shear mode in the phonon spectrum at the $\overline{\boldsymbol{I}}$ point of the Brillouin zone ('rigidity catastrophe'). Of course, any instability is predicted to occur at pressures larger than the amorphization pressure of $\alpha-\mathrm{SiO}_{2}$, which is quite common in first-order phase transitions somehow driven by (second-order-like) elastic or vibrational instabilities. Binggeli et al $[13,16]$ predict an elastic instability associated with 
decreasing acoustic velocities ( $B_{2}$ criterion). From their Brillouin spectroscopy study at high pressure, Gregoryanz et al [24] propose that this $B_{2}$ type instability is driven by the softening of $c_{44}$ with pressure. In contrast, Tse et al [14] predict a mechanical instability ( $B_{1}$ criterion) with a steep decrease of $c_{12}, c_{14}, c_{13}, c_{23}$ and $c_{33}$, this last modulus being the dominant factor. Chaplot et al [15] have found that the amorphization transition is initiated by a soft optic phonon at the Brillouin zone boundary rather than by a transverse acoustic mode near the zone center, the instability of the acoustic mode at $22 \mathrm{GPa}$ being related to the anomaly of the Born criterion $B_{1}$. Watson and Parker [17] propose a dynamical instability process in which the Born criteria are not involved. In their free energy calculations, de Boer et al [18] observe that the crystal is relatively weak in the $a$ direction, which favors an instability in the 110 direction, leading to a violation of the $B_{2}$ criterion. Finally, Choudhury et al [3] propose an amorphization transition related to the $B_{2}$ criterion. There is hence a considerable confusion concerning the mechanism of the amorphization process.

Although the pressure range required to clarify the amorphization process is still beyond reach for the ultrasonic technique, it is nevertheless clear [24, 29, 30] that uncertainties and contradictions in the pressure dependence of the elastic moduli driving the amorphization transition require new precise measurements of the elastic properties of $\alpha$-quartz as a function of pressure.

The purpose of this paper is to present experimental results, obtained by ultrasonic travel time measurements under hydrostatic pressure on single crystalline $\alpha$-quartz up to $1 \mathrm{GPa}$ at ambient temperature. In order to obtain reliable values for all the elastic moduli, we used a newly developed self-consistent numerical method [31], taking advantage of a redundant number of measurements of the travel time of the acoustic waves. By fitting simultaneously all the measured data, we are able to determine all components of the elastic compliance tensor and the piezoelectric contributions, from which the linear and volumic compressibilities are deduced, without any prerequisite. A special effort was made to eliminate the errors traditionally associated with pulse-echo overlap measurements. The treatment of the signal of the echoes has been performed with special care [32], enabling a highly accurate determination of the ultrasonic waves' transit time.

\section{Experimental procedure}

Two parallelepipedic samples were cut from the same single crystalline $\alpha-\mathrm{SiO}_{2}$ natural ingot of high purity. Their density $\left(\rho 2648.5 \mathrm{~kg} \mathrm{~m}^{-3}\right)$ at 1 bar and $298 \mathrm{~K}$ was found to be in agreement with the published value [1]. These two samples were oriented by x-ray Laüe diffraction. The $X, Y, Z$ axes of the first parallelepiped correspond to the $a, b^{*}$ and $c$ crystalline axes. The $X, Y^{\mathrm{t}}, Z^{\mathrm{t}}$ axes of the second one are obtained by a $45^{\circ}$ rotation of the $Y, Z$ axes around the $X$ axis. The perpendicularity and the parallelism between the faces of both samples were determined with a precision of a quarter of a degree and $1 \mu \mathrm{m}$ respectively. The dimensions of the samples were 7.543, 7.800 and $7.682 \mathrm{~mm}$ in the $X, Y$ and $Z$ directions respectively for the first sample and 7.125, 7.095, 8.269 $\mathrm{mm}$ in the $X, Y^{\mathrm{t}}$ and $Z^{\mathrm{t}}$ directions for the second one.

The ultrasonic system was a classical pulser receiver MATEC TB1000 setup. It sends a signal of adjustable frequency to a $4 \mathrm{~mm}$ diameter copper electrode in contact with the surface of the transducer to provide the excitation field. The same transducer is used for both sending and receiving the ultrasonic pulses. For ambient pressure experiments, we used $5 \mathrm{MHz}$ ceramic transducers for longitudinal and transverse waves and for high hydrostatic pressure experiments, 5 and $10 \mathrm{MHz} \mathrm{LiNbO}_{3}$ transducers with a diameter of $6 \mathrm{~mm}$ (Boston Piezo Inc. Optic). The transducer is bonded to the sample using a thin uniform acoustic coupling 
(SWC of Sofranel France). The high-pressure setup to measure the elastic properties of crystals was described in Hubert [32].

Hydrostatic pressure up to about $1 \mathrm{GPa}$ was applied in a piston-cylinder Basset hydraulic press $(\mathrm{P} 2)$ connected to a 'B18' pressure ${ }^{2}$ cell by a capillary tube. The pressure transmitting fluid was $99 \%$ pure pentane that remains fluid and hence produces hydrostatic pressures up to 1.2 GPa [33]. The pressure was measured using a manganin guage, whose resistance varies linearly with pressure $p(R)=k\left(R-R_{0}\right)$, where $R_{0}=120.035 Q$ and $k=0.33206 \mathrm{GPa} Q^{-1}$. The ultrasonic transit time was measured as a function of pressure using the ultrasonic pulseecho overlap technique. The sound velocity is given by $2 d(p) / t(p)$ where $d(p)$ is the length of the sample and $t(p)$ the transit time, both at pressure $p$. There is a difference between the transit times measured at ambient pressure in air and in the pressure transmitting medium. This difference can be explained by the change in frequency $(5-10 \mathrm{MHz})$ and in diameter $(8-$ $5 \mathrm{~mm}$ ) of the transducer between the ambient and high-pressure measurements. We did correct the results to take into account this effect. The transit times were measured both during the upstroke and the downstroke, in the five propagation directions mentioned previously, and for all the polarizations.

In a piezoelectric material the Christoffel equation of motion may be expressed as

$$
\rho v^{2} u_{i}=c_{i j k l}+\frac{e_{k j i} e_{j k l}}{E_{j k}^{s} n_{j} n_{k}} \quad n_{j} n_{k} u_{l},
$$

where $c_{i j k l}, e_{k j i}$ and $E^{s}{ }_{j k}$ are respectively the elastic compliances, the piezoelectric moduli and the dielectric constant upon constant strain. The solutions of this eigensystem give the velocities $v$ and the polarizations $u$ of the acoustic modes propagating along the direction $n$.

$\alpha$-quartz is a piezoelectric compound which crystallizes in the trigonal symmetry (space group $P 3_{12} 2$ ), therefore it has six independent elastic moduli, two piezoelectric and two dielectric constants. In the high symmetry directions $X, Y, Z, Y^{\mathrm{t}}$ and $Z^{\mathrm{t}}$, the eigenvalues of equation (3) are shown in table 1 . The various configurations are numbered from 1 to 15 , and these numbers will be used throughout this paper. From the table, it is seen that only two elastic constants can be directly derived from the experimental data i.e. $c_{33}$ and $c_{44}$ (longitudinal and transverse modes in the $Z$ direction, numbered as 7 and 8) and that the piezoelectric moduli appear only in the description of the waves polarized in the $X$ direction.

The 'classical' way of determining the elastic tensor is to make as many measurements as unknown independent values (eight here). Assuming $\theta$ is the standard error on the determination of $c_{33}$ or $c_{44}$ from experimental travel time values in the $Z$ direction (modes 7 and 8 ), the uncertainty on $c_{11}$ obtained from the travel time values of the quasilongitudinal and quasitransverse waves propagating in the $Y$ direction (modes 4 and 5) would be at least 3 (even if mode 1 is chosen and if $E^{\eta}$ is approximated by $E_{1} F^{T}$ ), 6 for $c_{14}$, and so forth. Moreover any mistake even made on only one experimental travel time determination will affect all the elastic moduli values. Obviously, any substitution method used to extract elastic coefficients from these equations would lead to a low precision result and the data consistency could not be checked. Consequently, the only way to prevent the error propagation in solving all these equations is to simultaneously determine all the elastic constants.

In order to determine the elastic moduli with the maximum precision and reliability, the transit times were measured in 15 configurations (five propagation directions and three

${ }^{2}$ It is a Marval 18 steel high-pressure cell. Its outer dimensions are $90 \mathrm{~mm} 90 \mathrm{~mm} \times 140 \mathrm{~mm}$. Its function is to generate the hydrostatic pressure on the sample, using liquid pentane as a pressure transmitting medium (fluid up to $1.5 \mathrm{GPa}$, i.e. $0.5 \mathrm{GPa}$ above the highest reached pressure). The maximum sample size with ultrasonic arrangement is roughly $2.5 \mathrm{~cm}^{-3}$. It was constructed by the Departement des Hautes Pressions de 1'Université Pierre et Marie Curie, Paris VI, France. 
Table 1. Effective second-order elastic moduli $c_{i j}$ of $\alpha$-quartz. $Y^{\mathrm{t}}$ and $Z^{\mathrm{t}}$ designate the propagation directions in the $(Y, Z)$ plane at an angle of $45^{\circ}$ to the $X$ axis. $e_{i \alpha}$ is the piezoelectric tensor and $E_{i j}^{s}$ is the effective dielectric tensor upon constant stress. $n$ propagation direction; $u$ polarization; longitudinal and quasilongitudinal ( $\mathrm{L}$ and $\mathrm{QL}$ ); transverse and quasitransverse ( $\mathrm{T}$ and $\mathrm{QT}$ ); fast and slow transverse (FT and ST). $E_{33}^{s}=E_{33}^{1} ; E_{11}^{s}=E_{11}^{T}-\left(e^{2} c_{44}+e^{2} c_{66}-2 e_{11} e_{14} c_{14}\right) /\left(c_{66} c_{44}-c^{2}\right)$.

\begin{tabular}{|c|c|c|c|c|}
\hline $\mathrm{Nr}$ & $n$ & $u$ & Mode & $\rho v^{2}$ \\
\hline 1 & $X$ & $X$ & $\mathrm{~L}$ & $c_{11}+e_{11}^{2}$ \\
\hline 2 & $X$ & $Z$ & FT & $c_{66}+c_{44}+\left(c_{66}-c_{44}\right)^{2}+4 c^{2}$ \\
\hline 3 & $X$ & $Y$ & ST & $c_{66}+c_{44}-y^{\left(c_{66}-c_{44}\right)^{2}+4 c^{2}}{ }^{2}$ \\
\hline 4 & $Y$ & $Y$ & QL & $c_{44}+c_{11}+\gamma^{\left(c_{44}-c_{11}\right)^{2}+4 c^{2}{ }_{14}^{2}}$ \\
\hline 5 & $Y$ & $Z$ & QT & $c_{44}+c_{11}-\left(c_{44}-c_{11}\right)^{2}+4 c^{2}{ }_{14}^{2}$ \\
\hline 6 & $Y$ & X & $\mathrm{T}$ & $c_{66}+\frac{e_{11}^{2}}{E_{11}^{s}}$ \\
\hline 7 & $Z$ & $Z$ & $\mathrm{~L}$ & $c_{33}$ \\
\hline 8,9 & $Z$ & $X, Y$ & $\mathrm{~T}$ & - \\
\hline 10 & $Y^{\mathrm{t}}$ & $Y^{\mathrm{t}}$ & QL & $c_{11}+c_{33}+2 c_{44}-2 c_{14}+{ }^{\mathbf{J}}\left(c_{11}-c_{33}-2 c_{14}\right)^{2}+4\left(c_{44}+c_{13}-c_{14}\right)^{2}{ }_{\mathbf{I}}^{4}$ \\
\hline 11 & $Y^{\mathrm{t}}$ & $Z^{\mathrm{t}}$ & QT & $\begin{array}{c}c_{11}+c_{33}+2 c_{44}-2 c_{14}-\overrightarrow{\left(c_{11}-c_{33}-2 c_{14}\right)^{2}+4\left(c_{44}+c_{13}-c_{14}\right)^{2}} 4 \\
\mathbf{I 1} \\
\mathbf{1}+\end{array}$ \\
\hline 12 & $Y^{\mathrm{t}}$ & $X$ & $\mathrm{~T}$ & $\left(c_{66}+c_{44}+2 c_{14} \begin{array}{c}(e 11+e 14)^{33} \\
\sim\left(E^{s}+E^{s}\right)\end{array}\right.$ \\
\hline 13 & $Z^{\mathrm{t}}$ & $Z^{\mathrm{t}}$ & QL & $c_{11}+c_{33}+2 c_{44}+2 c_{14}+\overline{\mathbf{J}}_{\left(c_{11}-c_{33}+2 c_{14}\right)^{2}+4\left(c_{44}+c_{13}-c_{14}\right)^{2}}^{1 \mathbf{T}}{ }^{\mathbf{1} 1}$ \\
\hline 14 & $Z^{\mathrm{t}}$ & $Y^{t}$ & QT & $\begin{array}{c}c_{11}+c_{33}+2 c_{44}-2 c_{14}-\overrightarrow{\left(c_{11}-c_{33}-2 c_{14}\right)^{2}+4\left(c_{44}+c_{13}-c_{14}\right)^{2}} 4 \\
\mathbf{I 1} \\
\mathbf{l}+\longrightarrow\end{array}$ \\
\hline 15 & $Z^{\mathrm{t}}$ & $X$ & $\mathrm{~T}$ & 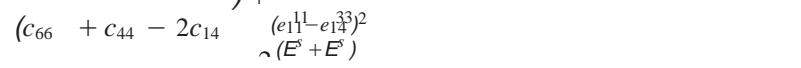 \\
\hline
\end{tabular}

polarizations) giving redundant results which have been simultaneously fitted to the equations presented in table 1 for every pressure.

Both non-linear least squares refinement by $X^{2}$ minimization and reverse Monte Carlo methods have been chosen to fit the equations shown in table 1 to experimental data. Computing methods have been extracted from 'Numerical Recipes' [34]. $X^{2}$ minimization is realized with a classical Levenberg-Marquardt algorithm [35, 36]. The 'mrqmin' routine used in the Levenberg-Marquardt algorithm requires knowledge of a one variable function $f\left(c_{i j}, e_{i \alpha}, E_{i j}^{T}\right)(x)$ and its derivatives versus all the fitted parameters $c_{i j}, e_{i \alpha}, E_{i j}^{I}$. In our case the $x$ variable represents the mode number and corresponds to one of the equations appearing in table 1 . The routine is very flexible and all parameters can be constrained or adjusted so that the influence of each parameter on the final result and on the fit quality can be tested. The code has been extensively and successfully tested [31] on results obtained on various samples of different point group symmetry but to be sure to escape from local minima, a more complex simulated annealing algorithm has also been introduced. This algorithm is based on a Monte Carlo method and is fully described in [37, 38]. In order to compare the results obtained by these two algorithms, the same input function described above for the Levenberg-Marquardt algorithm has been used. The quality of the Monte Carlo fitting procedure depends on the random number generator, the temperature step size, the number of iterations and the sampling scheme used for the parameters so that it has been used only to avoid local minima. 
In this process, in order to avoid non-linear effects in the minimized function, experimental travel times have been converted into $\rho v^{2}$ units using $\beta v^{2} 4 \rho d^{2} / t^{2}=C^{n}{ }_{u}$ and the known (or calculated by the previous iteration, see below) length and density of the sample. The term $C_{u}^{n}$ corresponds to the combinations of the elastic moduli presented in table 1 , where $n$ corresponds to the propagation direction and $u$ to the polarization (longitudinal and quasilongitudinal ( $\mathrm{L}$ and $\mathrm{QL}$ ); transverse and quasitransverse (T and QT); fast and slow transverse (FT and ST)). With uncertainties on time: $\delta t \sim 5 \mathrm{~ns}$ and on length: $\delta d 2 \mu \mathrm{m}$, the absolute typical error on 'experimental' $C_{u}^{n}$ is less than $1 \%$ but the relative error is smaller as it can be checked on the dispersion of the experimental results. Finally equations appearing in table 1 have been fitted to the experimental $\rho v^{2}$ by explicitly minimizing the quantity

$$
X^{2}=\sum_{\substack{i \\ i}} \frac{1}{\sigma_{i}^{2}}\left(f\left(c_{i j}, e_{i \alpha} E_{i j}^{T}\right)(x)-\rho v_{i}^{2}\right)^{2},
$$

where $\sigma_{i}$ is the uncertainty corresponding to the ' $i$ th' experimental measurement ' $\rho v^{2}$ ',

Supposing length and density are known at a given pressure $p$ all the elastic moduli can be deduced by ultrasonic measurements and therefore the volumic and linear compressibilities that are functions of the elastic constants only. Using these values, a first approximation of the length and of the density can be computed at a pressure $p_{+} \delta p$ for a small pressure increase $\delta p$. These values are then used to deduce the first approximation elastic moduli at the new pressure, obtained from the measurement of the transit time at $p+\delta p$ as described in the preceding paragraph. At this step, the density and length are respectively under- and overestimated, while the elastic moduli are underestimated. The values of the latter are then used as starting points in an iterative process, until convergence is reached. Since the variation of the sound velocity is mainly due to that of the elastic moduli, their dependence on length and density being only second order, this process is robust.

This method applied to deduce the elastic moduli from the transit time measurements in crystals of any symmetry is described in detail in a recent paper by Gauthier et al [31]. It does not require any $\mathrm{x}$-ray measurement for the pressure-induced length and density variation, provided one can neglect the difference between adiabatic and isothermal moduli. This approximation is justified in the case of $\alpha$-quartz where the difference is less than $1 \%$ [39]. Consequently, all components of the elastic compliance tensor including the piezoelectric contributions, from which the linear and volumic compressibilities are deduced, have been obtained from direct travel time measurements under pressure without any prerequisite.

\section{Results and discussion}

As explained in the preceding section, we measured the transit times $t$ as a function of pressure up to $1 \mathrm{GPa}$ in the five high-symmetry directions $X, Y, Z, Y^{\mathrm{t}}, Z^{\mathrm{t}}$, for the three polarizations. The relative variation with pressure of the 14 independent measurements (transverse modes are degenerate in the $Z$ direction) is shown in figures 1 (a)-(c) where the $t(p) t(p=0)$ have been normalized with respect to the transit times at ambient pressure.

Figure 1 summarizes the raw results obtained in the present study. The may be split in three groups showing common properties. Figure 1(a) gathers the propagation directions and polarizations where the propagation time decreases linearly with pressure. Figure 1(b) shows the pressure dependence of the transit time in the $X$ direction for the fast transverse mode (mode 2), in the $Y$ direction for the transverse mode (mode 6) and in the $Z^{\mathrm{t}}$ direction for the transverse mode (mode 15). The velocity of all these three modes decreases when the pressure is increased. This behavior has already been observed by McSkimin for modes 2 and 6. Mode 15 has never been investigated before. Finally, in figure 1(c) are gathered transit times with large 


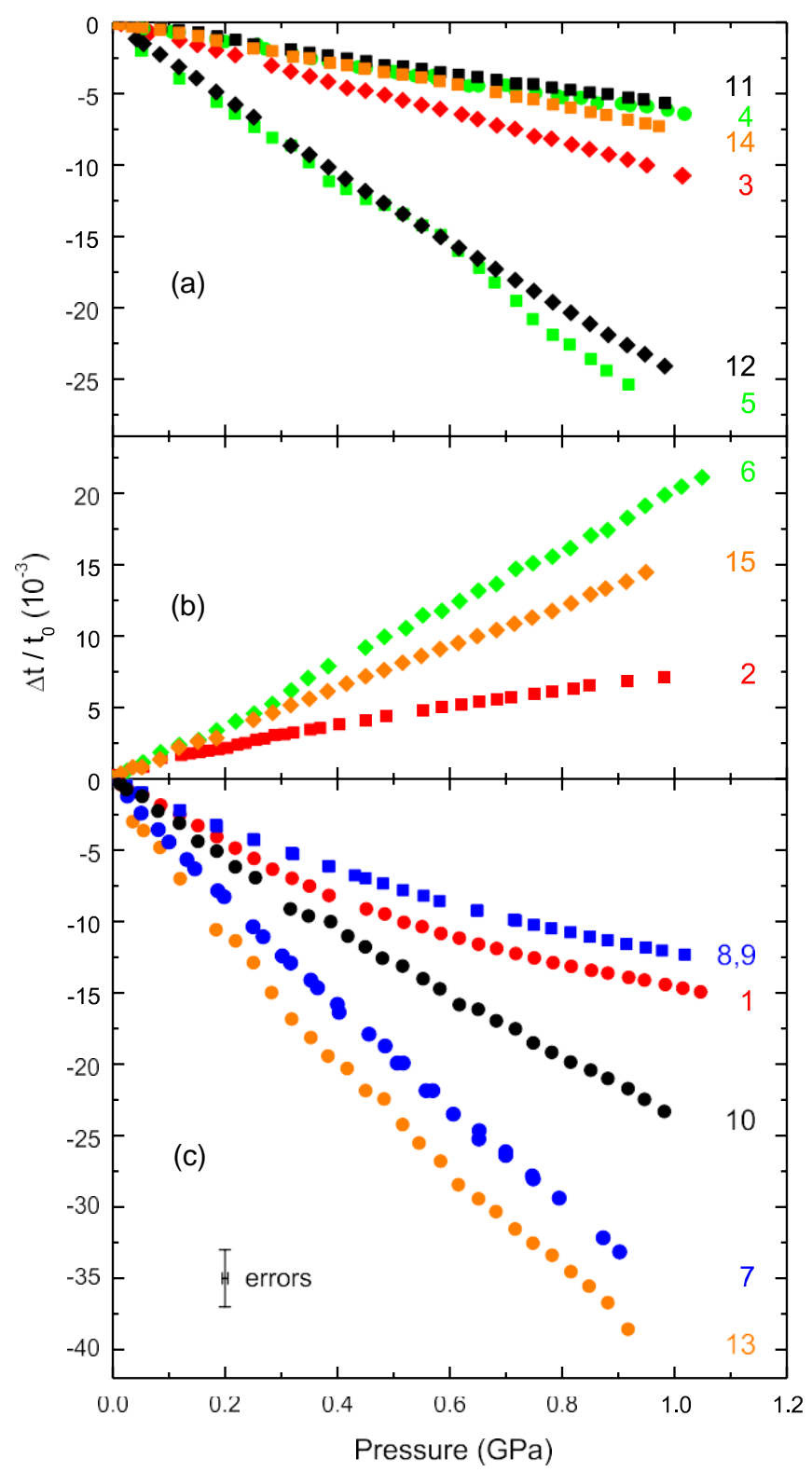

Figure 1. The relatives changes in transit times as a function of pressure of the 15 acoustic modes up to $1 \mathrm{GPa}$ for $\alpha-\mathrm{SiO}_{2}$ at $300 \mathrm{~K}$. Symbols are experimental data points: circles (longitudinal waves), squares and diamonds (fast and slow transverse waves). The numbers correspond to table 1 . The transit times increase with pressure for modes 2, 6 and 15 while the transit times for longitudinal waves in all directions (except $Y$ ) and the transverse waves in the $Z$ direction behave non-linearly with pressure. (Colors online correspond to different directions, $X$ red, $Y$ green, $Z$ blue, $Y^{\mathrm{t}}$ black and $Z^{\mathrm{t}}$ orange.)

non-linearities: mode 1 corresponds to the longitudinal mode propagating in the $X$ direction, mode 7 is the longitudinal mode in $Z$ direction, modes 8 and 9 are the degenerate transverse modes propagating in the $Z$ direction, modes 10 and 13 are the quasilongitudinal modes propagating in the $Y^{\mathrm{t}}$ and $Z^{\mathrm{t}}$ directions. Such a low-pressure non-linearity has already been 


\begin{tabular}{lllllll}
\hline \multicolumn{7}{c}{ Table 2. Second-order elastic constants (in GPa) and their pressure derivatives at ambient pressure } \\
\\
and temperature.
\end{tabular}

observed in compounds of the berlinite family, isoelectronic to $\alpha$-quartz [40-42]. Moreover, it is by no way in contradiction with the results of McSkimin [29]. The narrow pressure range over which this author made his measurements (30000 psi-0.2 GPa) did not allow the observation of a non-linear behavior of the elastic properties.

In order to have an easier comparison between our results and those of the literature, especially those obtained by Brillouin scattering, it is more convenient to convert the transit times into elastic moduli. This has been done using the method exposed in the preceding section. As seen in table 1, there are four piezoelectric modes, (1, 6, 12 and 15). Unfortunately, modes 1 and 6 involve the same piezoelectric parameter $\left(e^{2} / E_{11}^{s}\right)$. In other words, four

quantities $e_{11}, e_{14}, E^{\mathrm{T}}{ }_{11}$ and $E_{33}^{\mathrm{T}}$ have to be determined from only three equations. Since the pressure dependence of the dielectric constants of quartz is not known, we have supposed as a first approximation, that they do not depend on pressure and are fixed to the ambient condition values, i.e. $\left(E_{1}^{\mathrm{T}} / E_{0}\right)=4.52$ and $\left(E_{33}^{\mathrm{T}} / E_{0}\right)=4.64[43]$.

The values of the elastic constants at ambient conditions and their pressure dependence deduced from our measurements are reported in figure 2. They are summarized in table 2 and compared with those obtained by ultrasonic [29, 30], Brillouin scattering [24] and calculations $[3,18]$. There is a relatively good agreement between all the ultrasonic results. Only the sign of $c_{14}$ and the value of $c_{12}$ obtained by Wang et al [30] do not agree with the other results. It is highly probable that the explanation for this discrepancy lies in a confusion between $Y^{\mathrm{t}}$ and $Z^{\mathrm{t}}$ axes, confusion that also affects all other results. In the same table, the pressure derivatives of all the elastic constants are compared with the published results. There is again a good agreement between the ultrasonic measurements, except for the derivative of $c_{14}$ obtained by Wang [30] for the already mentioned reason. The small discrepancies compared to McSkimin et al [29] may arise from the fact that they did not measure the pressure dependence of modes 12,13, 14 and 15 under pressure and consequently their precision in the determination of the variation of $c_{13}$ (obtained with no redundancy) and even $c_{33}$ is rather low and may influence the other pressure derivatives.

On the other hand, there is typically a difference from 1 to $15 \%$ between the ultrasonic and the Brillouin scattering results. The difference is particularly large for $c_{11}, c_{33}$ and $c_{13}$. The discrepancy is much larger for the pressure derivatives of the elastic constants. In particular, $c_{12}$ is found to be pressure independent and the derivative of $c_{44}$ has a negative sign in this last experiment. We suspect that an error occurred during the extraction of the $c_{i j}$ from the Brillouin 

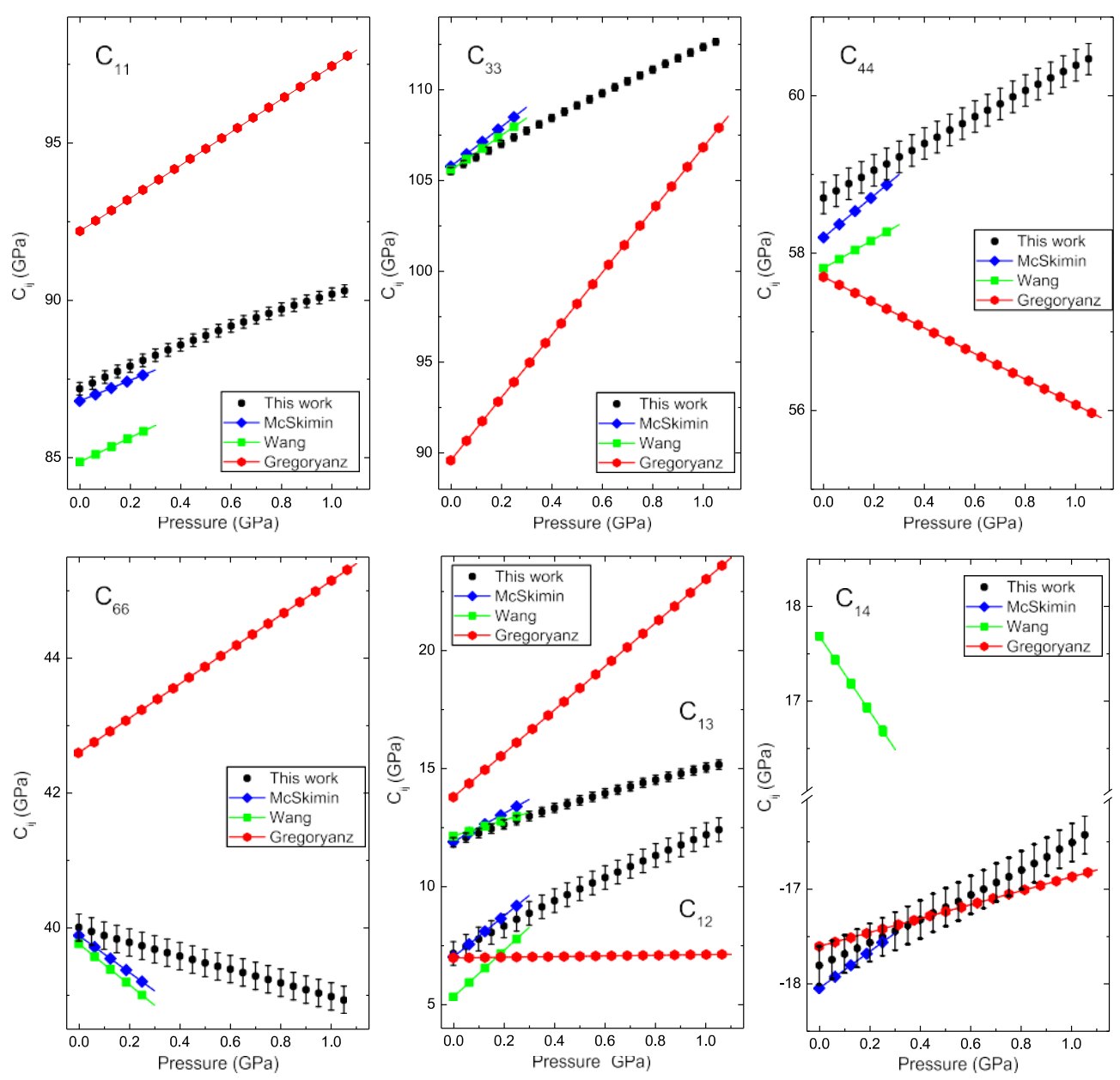

Figure 2. Pressure dependence of the elastic constants $c_{i j}$ (black circles) compared with those of McSkimin et al [29] ((blue) line with diamonds), Wang et al [30] ((green) line with squares) and Gregoryanz et al [24] ((red) line with hexagons). The large discrepancies between the results of Gregoryanz [24] and the other authors (large difference in the values, and in the sign of $\partial c_{66} / \partial p$, $\partial c_{44} / \partial p$ ) may be due to an error occurred during the extraction of the $c_{i j}$ from the Brillouin shifts measurements, in such a way that $c_{44}$ and $c_{66}$ were inverted. For Wang et al's results [30] the difference in sign of $c_{14}$ and of $\partial c_{14} / \partial p$ probably lies in a confusion between the $Y^{\mathrm{t}}$ and $Z^{\mathrm{t}}$ axis.

shift measurements, in such a way that $c_{44}$ and $c_{66}$ were inverted, with all the associated consequences on the determination of the other elastic constants. This has consequences on the hypothesis made by these authors on the pressure-induced destabilization process of $\alpha$ quartz; hence the destabilization process that they proposed via the softening of $c_{44}$ cannot be considered any more.

The method we used to deduce the elastic moduli from the travel time enabled us also to determine the piezoelectric constants, provided one remembers that the pressure variations of the dielectric constants upon constant stress have been neglected. The results are shown in table 3. We have found a non-linear variation of both piezoelectric parameters, but much more pronounced for $e_{14}$ than for $e_{11}$. Obviously these determinations suffer from our hypothesis on the pressure variation of the dielectric tensor $E^{\mathrm{T}}$ but the general tendencies may be used 


\begin{tabular}{|c|c|c|c|c|c|c|c|c|c|c|}
\hline \multirow{3}{*}{$\begin{array}{l}\text { Ref. } \\
\text { This work }\end{array}$} & \multicolumn{10}{|c|}{$\begin{array}{l}\text { Table 3. Values of the dielectric and piezoelectric constants at room pressure and temperature. } \\
e_{i \alpha} \text { in } \mathrm{C} \mathrm{m}^{-2} \text { and } E_{i j} \text { in } \mathrm{F} \mathrm{m}^{-1}, p \text { in GPa. } e_{1}=e^{2} / E_{1}^{s}, e_{7}=\left(e_{11}+e_{14}\right)^{2} / 2\left(E^{s}+E_{11}^{s}\right) \text {, } \\
e_{3}=\left(e_{11}-e_{14}\right)^{2} / 2\left(E^{s}{ }_{11} E^{s}\right)_{33} \text { The asterisks denote the values calculated by us using } \partial e_{11} / \partial p= \\
\left(\partial e_{11} / \partial \eta_{1}\right) / B_{a} \text {. }\end{array}$} \\
\hline & \multicolumn{10}{|c|}{$e_{11} e_{14} \partial e_{11} / \partial p \partial e_{14} / \partial p e_{1} e_{2} e_{3} \partial e_{1} / \partial p \partial e_{2} / \partial p \partial e_{3} / \partial p$} \\
\hline & 0.17 & -0.04 & -0.03 & 0.3 & 0.75 & 0.28 & 0.11 & -0.2 & -0.8 & 0.6 \\
\hline McSkimin [29] & 0.171 & -0.041 & - & - & 0.77 & - & - & 0.005 & - & - \\
\hline Wang [30] & - & - & - & - & - & - & - & 2.32 & - & -0.37 \\
\hline Graham [44] & \multicolumn{3}{|c|}{$0.1711-0.0406-0.026^{*}$} & - & - & - & - & - & - & - \\
\hline
\end{tabular}

with confidence. These results demonstrate the continuous decrease under pressure of the piezoelectric effect associated with $e_{11}$, in agreement with the data obtained by Graham [44] through shock wave experiments. From his result $\partial e_{11} / \partial \eta_{11} 2.64 \mathrm{Cm}^{-2}$ where $\eta_{1}$ is the strain tensor we get $\partial e_{11} / \partial p=-0.026 \mathrm{C} \mathrm{m}^{-2} \mathrm{GPa}^{-1}$ (using $\partial e_{11} / \partial p=\left(\partial e_{11} / \partial \eta_{1}\right) / B_{a}$ ) which is close to our value $-0.03 \pm 0.01 \mathrm{C} \mathrm{m}^{-2} \mathrm{GPa}^{-1}$. To our knowledge we present here the first results on the pressure variation of $e_{14}$, results which should be confirmed [45]. According to our results the piezoelectric coupling coefficient decreases from $9.2 \%$ to $7.2 \%$ at $1 \mathrm{GPa}$ in the $X$ direction and from $13.5 \%$ to $11.0 \%$ in the $Z$ direction. At the same pressure, the piezoelectric effect nearly vanishes in the $Z^{\mathrm{t}}$ direction whereas the piezoelectric coupling coefficient increases from $5.8 \%$ to $11.2 \%$ in the $Y^{\mathrm{t}}$ direction. This complete determination of the variation of the piezoelectric effect under pressure enables a very accurate determination of the elastic constants.

From all these results it appears that only the shear elastic stiffness modulus $c_{66}$ of $\alpha$-quartz shows unusual pressure dependence with a negative value for $\partial c_{66} / \partial p$, behavior also found in the berlinite $\mathrm{AlPO}_{4}$ [40]. As seen in the introduction, a pressure-induced softening of an elastic modulus may indicate incipient lattice instability because it corresponds to a reduction of the crystal stiffness for the associated acoustic-phonon mode but $c_{66}$ (and $c_{44}$ ) does not correspond in a trigonal system to any particular mechanical instability.

Figure 3 shows the variation of the linear and bulk moduli $B_{a}, B_{c}$ and $B_{0}$ versus pressure. Their ambient pressure values and their derivatives are summarized in table 4 together with published results. The values obtained for these moduli at ambient pressure are in good agreement with the values of the literature [7, 29, 30], even with values computed by us from $x-$ ray data [11] except for Gregoryanz's results for $B_{c}$ [24]. On the contrary, there is a difference in the value of $\partial B_{0} / \partial p$, which is smaller than the published values. This difference of $25 \%$ can be explained by the difference in the determination method for the elastic constants, and in the pressure range over which the measurements were performed. The values at ambient conditions are in general easy to check through several experiments. However, as explained in the experimental section, the standard way to determine the elastic constants as a function of pressure is to perform just as many measurements as unknown parameters. In such a method, there is a propagation of the errors, which increases the inaccuracy of the results. Using the method presented in [31] gives roughly the same precision on all the elastic moduli, and increases therefore the accuracy of the bulk modulus pressure derivative to few per cent. As already mentioned, McSkimin et al [29] did not make any measurement in the $Z^{\mathrm{t}}$ direction and only two in the $Y^{\mathrm{t}}$ direction under pressure, which explains the difference in $B_{c}^{\mathrm{t}}$. In conclusion, it can be clearly seen in figure 3 that the bulk and the linear moduli all increase with pressure but that their pressure derivatives are not constants.

From the pressure dependence of the elastic moduli, one can also determine the Born criteria, from which the stability of the crystalline structure may be discussed. The criteria 

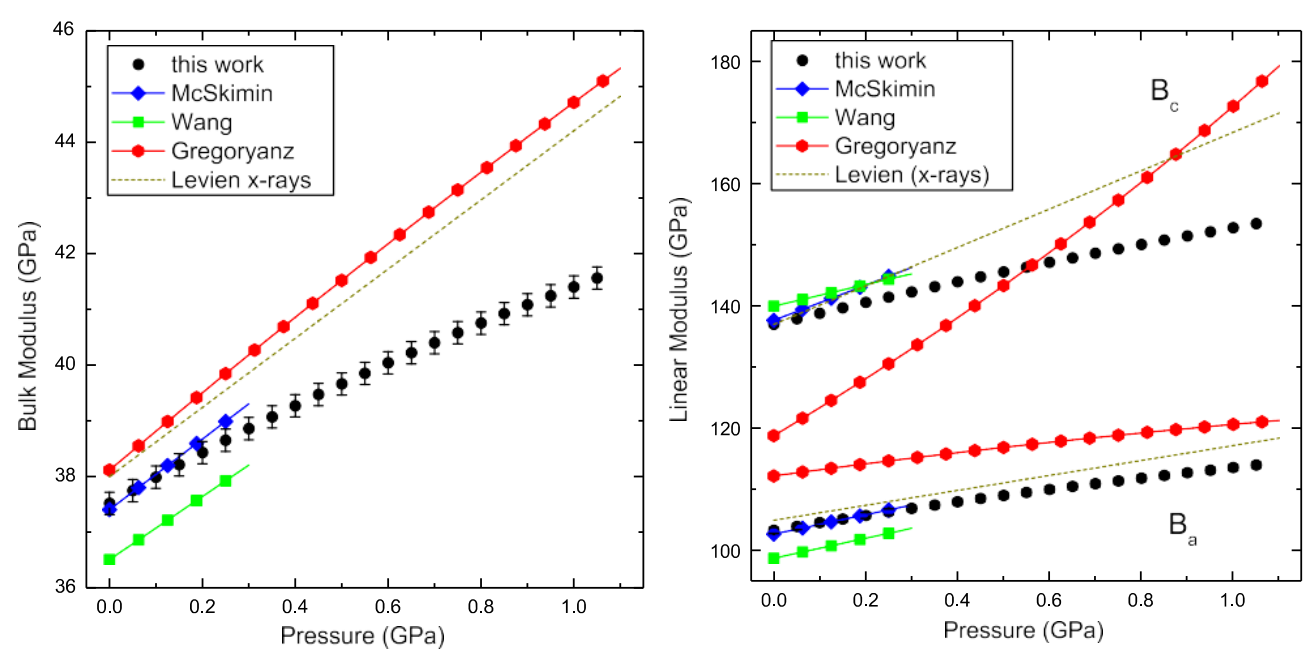

Figure 3. Variation of the linear and bulk modulus of $\alpha-\mathrm{SiO}_{2}$ at $300 \mathrm{~K}$ versus pressure. The dotted lines have been computed by us from x-ray data using Murnaghan's equation of state. The $B_{c}$ values by Gregoryanz [24] are never within the error bars.

Table 4. Linear moduli, bulk modulus and their pressure derivatives for $\alpha-\mathrm{SiO}_{2}$ and the pressure domain used for their determination. The asterisks denote the values calculated by us.

\begin{tabular}{lllllllll}
\hline \multirow{2}{*}{ Ref. } & This work & $\begin{array}{l}\text { McSkimin } \\
{[29]}\end{array}$ & $\begin{array}{l}\text { Wang } \\
{[30]}\end{array}$ & $\begin{array}{l}\text { Gregoryanz } \\
{[24]}\end{array}$ & $\begin{array}{l}\text { Kim } \\
{[7]}\end{array}$ & $\begin{array}{l}\text { Levien } \\
{[11]}\end{array}$ & $\begin{array}{l}\text { Choudhury } \\
{[3]}\end{array}$ & $\begin{array}{l}\text { de Boer } \\
{[18]}\end{array}$ \\
\hline Technique & US & US & US & Brillouin & X-ray & X-ray & Calcul. & Calcul. \\
$p_{\max }(\mathrm{GPa})$ & $\approx 1$ & $\approx 0.2$ & $\approx 0.2$ & 20 & 24 & $\approx 6$ & 38 & 24 \\
$B_{0}(\mathrm{GPa})$ & $37.5(0.2)$ & 37.4 & 36.5 & 36.16 & 38.7 & 38 & $38.6^{*}$ & $38.4^{*}$ \\
$B_{a}(\mathrm{GPa})$ & $103.3(0.5)$ & $102.7^{*}$ & $98.8^{*}$ & $112.2^{*}$ & - & $105^{*}$ & $103.6^{*}$ & $109.4^{*}$ \\
$B_{c}(\mathrm{GPa})$ & $137.0(0.5)$ & $137.7^{*}$ & $140.0^{*}$ & $118.9^{*}$ & - & $137^{*}$ & $151.4^{*}$ & $128.8^{*}$ \\
$\partial B_{0} / \partial p$ & $4.7(0.5)$ & 6.3 & 5.6 & $6.5^{*}$ & 5.2 & 6.2 & - & $4.7^{*}$ \\
$\partial B_{a} / \partial p$ & $12.4(0.5)$ & $15.9^{*}$ & $16.3^{*}$ & $10.2^{*}$ & - & $12.2^{*}$ & - & $10^{*}$ \\
$\partial B_{c} / \partial p$ & $18.5(0.5)$ & $28.3^{*}$ & $17.6^{*}$ & $43.8^{*}$ & - & $31.4^{*}$ & - & $25.6^{*}$ \\
\hline
\end{tabular}

are given in equations $(1 a)$ and $(1 b) . B_{1}$ is related to the bulk and linear moduli, and $B_{2}=$ $2\left(c_{66} C_{44} \epsilon^{2}\right) P_{4} 0$ is a combination of moduli related to transverse acoustic phonons [26-28]. In figure 4 , we have plotted the pressure dependence of both criteria, measured up to $1 \mathrm{GPa}$, together with the results obtained by other authors. The dashed lines correspond to a linear extrapolation of our low-pressure data. The main finding of these results is the strong nonlinearity of the $B_{1}$ criterion pressure dependence whereas $B_{2}$ behaves in a very smooth way.

The precision of our results enables us to extrapolate the Born criteria to high pressure, using a quadratic form. Within this extremely crude extrapolation, $B_{2}$ increases continuously as expected and $B_{1}$ presents a maximum in disagreement with previously published data $[3,13,16,18,24]$. It is clear that the pressure range explored in the present experiments is much too narrow to give a sufficient accuracy to this extrapolation, but it shows nevertheless that an accurate determination of the elastic moduli and their pressure dependence is sufficient to give a correct tendency. The fact that the destabilization process in quartz is related to the $B_{1}$ Born criterion, and not to $B_{2}$, means that the driving mechanism is not a shear stress, but is a volumic process. This is in agreement with recent abinitio calculations by 

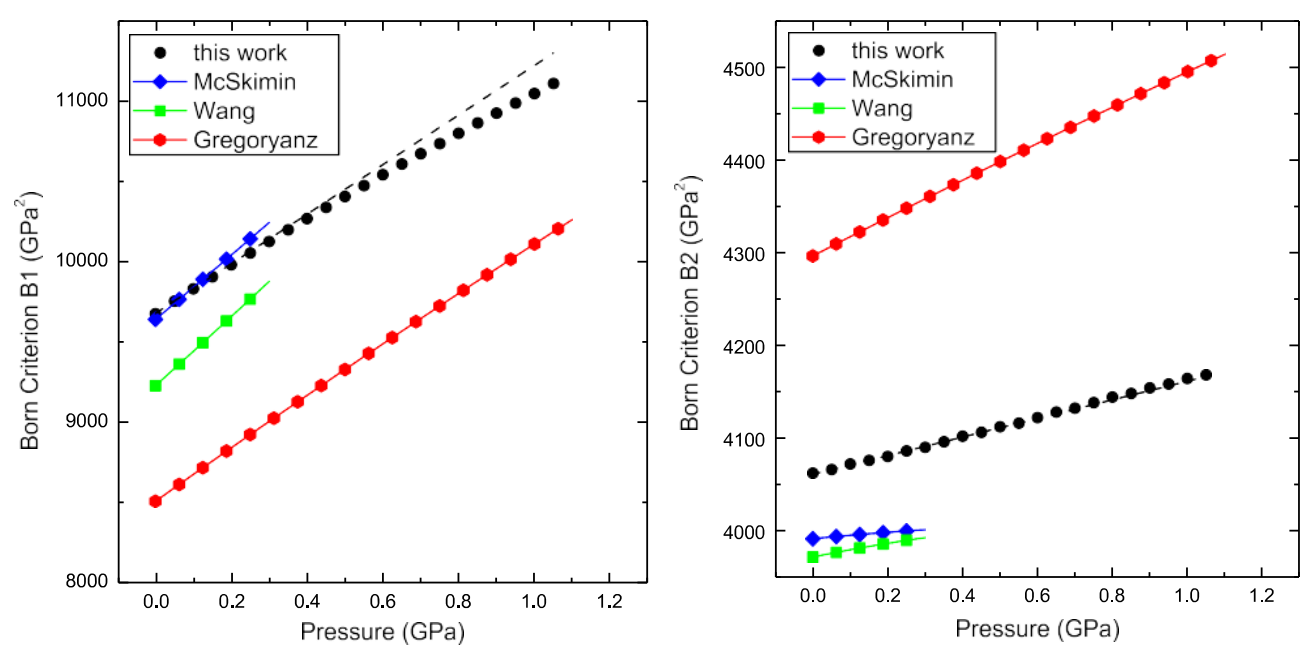

Figure 4. Variation of the Born criterion of $\alpha-\mathrm{SiO}_{2}$ at $300 \mathrm{~K}$ versus pressure. Dashed lines correspond to a linear extrapolation of low-pressure data.

Baroni et al [46]. Measurements using the Paris-Edinburgh press are under way to expand the explored pressure range and reach the point where $B_{1}$ is expected to present a maximum.

\section{Conclusion}

Using the ultrasonic pulse-echo technique and a newly developed method to treat the data, all the elastic moduli of $\alpha$-quartz have been obtained as a function of pressure up to $1 \mathrm{GPa}$ with a high accuracy. The pressure derivatives of $c_{14}$ and $c_{44}$ are positive and only that of $c_{66}$ is negative. Therefore the elastic constants and their pressure derivatives behave like those of the berlinite $\mathrm{AlPO}_{4}$, but $\alpha$-quartz is stiffer than berlinite. The deduced values at ambient conditions of the linear and bulk moduli are in good agreement with published values. However, we find a clear discrepancy with the published values for their pressure derivative.

The measured piezoelectric coefficients are consistent with those reported previously at zero pressure and their pressure dependence has been determined up to $1 \mathrm{GPa}$. It should be emphasized that it is the first complete determination of the pressure dependence of the piezoelectricity, to the best of our knowledge. The pressure derivative of $e_{14}$ is positive whereas that of $e_{11}$ is negative so the piezoelectric effect associated with $e_{11}$ decreases with pressure whereas the effect associated with $e_{14}$ increases.

Finally the two stability conditions (Born criteria) $B_{1}$ and $B_{2}$ have been calculated from our determination of the elastic tensor. It is shown that the $B_{1}$ condition presents a large nonlinearity from which it may be concluded, from a very crude extrapolation of the criteria at high pressure, that the pressure-induced amorphization is closely related to this 'volumic' criterion, and not to an acoustic soft mode behavior as has often been proposed. Nevertheless, our understanding of the thermodynamic factors that influence the amorphization of quartz still remains incomplete.

\section{Acknowledgments}

We wish to acknowledge Michèle Jacquet for help during the orientation of the samples by x-ray Laüe diffraction and Stefan Klotz for careful reading of the manuscript. 


\section{References}

[1] Bottom V 1922 Introduction to Quartz Crystal Unit Design (New York: Van Nostrand)

[2] Sharma S and Sikka S 1996 Prog. Mater. Sci. 401

[3] Choudhury N and Chaplot S 2006 Phys. Rev. B 73094304 and references therein

[4] Page Y and Donnay G 1967 Acta Crystallogr. B 322456

[5] Lager G, Jorgensen J and Rotella F 1982 J. Appl. Phys. 536751

[6] Kihara K 1990 Eur. J. Mineral. 263

[7] Kim-Zajonz J, Werner S and Schulz H 1999 Z. Kristallogr. 214324

[8] Kingma K, Hemley R, Mao H and Vleben D 1993 Phys. Rev. Lett. 703927

[9] Kingma K, Mao H K and Hemley R 1996 High Pressure Res. 14363

[10] Hazen R, Finger L, Hemley R and Mao H 1989 Solid State Commun. 72507

[11] Levien L, Prewitt C and Weidner D 1980 Am. Mineral. 65920

[12] Chelokowski J, Troullier N, Martins J and King H 1991 Phys. Rev. B 44489

[13] Binggeli N and Chelikowsky J 1992 Phys. Rev. Lett. 692220

[14] Tse J and Klug D 1991 Phys. Rev. Lett. 673559

[15] Chaplot S and Sikka S 1994 Proc. XIII AIRAPT Int. Conf. (Bangalore, 1991) ed A K Singh (New Delhi: Oxford and IBH Publ. Co.) p 25

[16] Binggeli N, Keskar N and Chelikowsky J 1994 Phys. Rev. B 493075

[17] Watson G and Parker S 1995 Phil. Mag. Lett. 7159

[18] de Boer K, Jansen A, van Santen R, Watson G and Parker S 1996 Phys. Rev. B 54826

[19] Toledano P and Machon D 2005 Phys. Rev. B 71024210

[20] McNeil L and Grimsditch M 1992 Phys. Rev. Lett. 6883

[21] Serghiou G, Winter R and Hammack W 1992 Phys. Rev. Lett. 683311

[22] Hemmati M, Chizmeshya A, Wolf G, Poole P, Shao J and Angell A 1995 Phys. Rev. B 5114841

[23] Murashov V 1996 Phys. Rev. B 53107

[24] Gregoryanz E, Hemley R, Mao H and Gillet P 2000 Phys. Rev. Lett. 843117

[25] Born M and Huang K 1954 Dynamical Theory of Crystal Lattices (London: Oxford University Press)

[26] Carpenter M and Salje E 1998 Eur. J. Mineral. 10693

[27] Terhune R, Kushida T and Ford G 1985 Phys. Rev. B 328416

[28] Bulou A 1992 J. Physique I 21445

[29] McSkimin H, Andreatch P and Thurston R 1965 J. Appl. Phys. 361624

[30] Wang Q, Saunders G, Lambson E, Tschaufeser P, Parker S and James B 1992 Phys. Rev. B 4510242

[31] Gauthier M, Hubert C and Decremps F 2007 unpublished

[32] Hubert C 2005 Thèse Université Pierre et Marie Curie-Paris 6 (unpublished) Nataf L, Decremps F, Gauthier M and Syfosse G 2006 Ultrasonics 44 e555

[33] Klotz S, Philippe J and Cochard E 2006 J. Phys. D: Appl. Phys. 391674

[34] Press W H, Teukolsky S A, Vetterling W T and Flannery B P 2002 Numerical Recipes in C $++2 \mathrm{nd} \mathrm{edn}$ (Cambridge: Cambridge University Press)

[35] Press W H, Teukolsky S A, Vetterling W T and Flannery B P 2002 Numerical Recipes in C $++2 \mathrm{nd} \mathrm{edn}$ (Cambridge: Cambridge University Press) chapter 15, p 688

[36] Vetterling W T, Teukolsky S A, Press W H and Flannery B P 2002 Numerical Recipes, Example Book (C++) 2nd edn (Cambridge: Cambridge University Press) chapter 15, p 252

[37] Press W H, Teukolsky S A, Vetterling W T and Flannery B P. 2002 Numerical Recipes in C $++2 \mathrm{nd}$ edn (Cambridge: Cambridge University Press) chapter 10, p 455

[38] Vetterling W T, Teukolsky S A, Press W H and Flannery B P 2002 Numerical Recipes, Example Book (C++) (Cambridge: Cambridge University Press) chapter 10, p 181

[39] Barron T and Pasternak A 1987 J. Phys. C: Solid State Phys. 20215

[40] Sidek H, Saunders G, Hong W, Bin X and Jianru H 1987 Phys. Rev. B 367612

[41] Hankey R and Schuele E 1970 J. Acoust. Soc. Am. 48190

[42] Palmier D 1996 Thèse Université Montpellier II (unpublished)

[43] Bechman R 1958 Phys. Rev. 1101060

[44] Graham R 1972 Phys. Rev. B 64779

[45] Calderon E, Gauthier M, Decremps F and Polian A 2007 unpublished

[46] Baroni S, Gironcoli S, Corso A D and Giannozzi P 2001 Rev. Mod. Phys. 73515 